\title{
The nonlinear integro-differential Ito dynamical equation via three modified mathematical methods and its analytical solutions
}

https://doi.org/10.1515/phys-2020-0004

Received Apr 24, 2018; accepted Jan 22, 2020

\begin{abstract}
In this work, we construct traveling wave solutions of (1+1) - dimensional Ito integro-differential equation via three analytical modified mathematical methods. We have also compared our achieved results with other different articles. Portrayed of some 2D and 3D figures via Mathematica software demonstrates to understand the physical phenomena of the nonlinear wave model. These methods are powerful mathematical tools for obtaining exact solutions of nonlinear evolution equations and can be also applied to non-integrable equations as well as integrable ones. Hence worked-out results ascertained suggested that employed techniques best to deal NLEEs.
\end{abstract}

Keywords: Integro-differential Ito equation, Generalized direct algebraic method, Extended simple equation method, Modified F-expansion method

\section{Introduction}

The world around us is basically nonlinear. In this regards nonlinear partial differential equations (NPDEs) are main significance to describe the complex physical phenomena; for example, nonlinear wave propagation can occur in the scopes of elasticity theory, fluid dynamics, plasma physics, and nonlinear optics.The exploration of analytical, exact solutions for NPDEs has become quite prominent due to the recently great advances gained in the computational techniques.Several efficient and powerful methods can be

*Corresponding Author: Aly Seadawy: Mathematics Department, Faculty of Science, Taibah University, Al-Madinah Al-Munawarah, Saudi Arabia; Email: Aly742001@yahoo.com

Asghar Ali: Mathematics Department, University of Education, Lahore, Pakistan

Noufe Aljahdaly: Department of Mathematics, Faculty of science and arts - Rabigh campus, King Abdulaziz University, Jeddah, Saudi Arabia

๖ Open Access. @ 2020 A. Seadawy et al., published by De Gruyter.

(cc) BY 4.0 License applied for finding the analytical solutions such as; Ricatti Bernoulli's sub-ODE method [1, 2], Modified extended direct algebraic method $[3,4,6]$, the homogeneous balance method, the modified simple equation method [7-9], auxiliary equation method [10], the modified extended mapping method [11-14], extended Jacobian elliptic function expansion method, the modified extended tanh-function method, the generalized Kudryashov method, the sinecosine method [15], the Hirota's bilinear method [16], Darboux transformation $[17,18]$, semi-inverse variational principle [19], the hyperbolic tangent expansion method [20], the inverse scattering transform [21], the tanhsech method and the extended tanhcoth method, the first integral method [22], the symmetry method, the soliton ansatz methods [23-35, 38].

Article purpose is to investigate exact solutions of integro-differential Ito equation by employing the three analytical modified mathematical methods. The integrodifferential Ito equation having fruitful applications in mathematical physics.In previous authors [39, 40] applied generalized Kudryashov and $\left(G^{\prime} / G, 1 / G\right)$ methods respectively for exact traveling wave solutions for Eq. (10). But the aspire our presented work is that, we give concentration for finding analytical solutions of Eq. (10) by generalized direct algebraic, extended simple equation and modified Fexpansion methods. The derived solutions are productive tools for solving numerous problems in the field applied sciences.

The reminant article arranged sections (2-5) as, Description of proposed steps in 2, apply methods in 3. Results discussion in 4 and Summary in 5.

\section{Description proposed methods:}

Consider

$$
P_{1}\left(v, v_{x}, v_{t}, v_{x x}, v_{t t}, v_{x t}, \ldots\right)=0
$$

Let

$$
v=V(\xi), \quad \xi=x-\omega t,
$$


Put (2) in (1),

$$
P_{2}\left(V, V^{\prime}, V^{\prime \prime}, \ldots\right)=0
$$

\subsection{Generalized Direct Algebraic Method:}

Let solution (3) has,

$$
\begin{aligned}
V & =\sum_{i=0}^{n} A_{i} \Psi^{i}+\sum_{i=-1}^{-n} B_{-i} \Psi^{i}+\sum_{i=2}^{n} C_{i} \Psi^{i-2} \Psi^{\prime} \\
& +\sum_{i=1}^{n} D_{i}\left(\frac{\Psi^{\prime}}{\Psi}\right)^{i}
\end{aligned}
$$

Suppose $\Psi$ satisfies following,

$$
\Psi^{\prime}=\sqrt{r_{1} \Psi^{2}+r_{2} \Psi^{3}+r_{3} \Psi^{4}}
$$

where $r_{1}, r_{2}, r_{3}$ are arbitrary constants.

Put (4) with (5) in (3), attained system of collection containing $\omega, r_{1}, r_{2}$ and $r_{3}$. Putting these values with solution $\Psi$ in (4), achieved the require destination of (1).

\subsection{Extended Simple Equation Method}

Let (3) has solution,

$$
V=\sum_{i=-n}^{n} A_{i} \Psi^{i}
$$

Let $\Psi$ gratify,

$$
\Psi^{\prime}=l_{0}+l_{1} \Psi+l_{2} \Psi^{2}+l_{3} \Psi^{3}
$$

Substituting (6) along with (7) into (3). After solving, transfer obtained values of the parameters and solution of $\Psi$ into (7). We obtained solution of (1).

\subsection{Modified F-expansion Method:}

Step 1: Let us suppose that (3) has solution as:

$$
V=a_{0}+\sum_{i=1}^{n} a_{i} F^{i}(\xi)+\sum_{i=1}^{n} b_{i} F^{-i}(\xi)
$$

Let $F$ gratifies,

$$
F^{\prime}=A+B F+C F^{2} .
$$

Step 2: Put (10) along (11) in (3), solving for require parameters values.

Step 3: Selective values $C, B, A$ and $F$ from Table 1 [41] and substitute $a_{i} b_{i}$ into Eq. (5), completed for solution (1).

\section{Applications:}

\subsection{Application of Generalized Direct Algebraic Method:}

Consider integro-differential Ito equation [39, 40],

$$
u_{t t}+u_{x x x t}+3\left(2 u_{x} u_{t}+u u_{x t}\right)+3 u_{x x} \partial_{x}^{-1}\left(u_{t}\right)=0 .
$$

Let

$$
u(x, t)=v_{x}(x, t), \xi=x-\omega t,
$$

Putting (11) in (10),twice integrate and integration constant, yields

$$
\omega v^{\prime}-v^{\prime \prime \prime}-3\left(v^{\prime}\right)^{2}=0
$$

Let (12) has solution,

$$
v(\xi)=A_{0}+A_{1} \Psi+\frac{B_{1}}{\Psi}+D_{1} \frac{\Psi^{\prime}}{\Psi}
$$

Put (13) along with (5) in (12), after solving, we have

$$
A_{1}= \pm \sqrt{r_{3}}, \quad D_{1}=-1, \quad B_{1}=0, \quad \omega=r_{1}
$$

Put (14) in (13), we have

\section{Case - I}

$$
\begin{aligned}
v_{1} & =A_{0}-\frac{\sqrt{r_{3}}\left(r_{1}\left(\epsilon \operatorname{coth}\left(\frac{1}{2}\left(\xi+\xi_{0}\right) \sqrt{r_{1}}\right)+1\right)\right)}{r_{2}} \\
& -\frac{r_{1}^{3 / 2} \epsilon \operatorname{csch}^{2}\left(\frac{1}{2}\left(\xi+\xi_{0}\right) \sqrt{r_{1}}\right)}{\frac{\left(2 r_{2}\right)\left(-r_{1}\left(\epsilon \operatorname{coth}\left(\frac{1}{2}\left(\xi+\xi_{0}\right) \sqrt{r_{1}}\right)+1\right)\right)}{r_{2}}},
\end{aligned}
$$

$$
\begin{aligned}
u_{1} & =\frac{\left(\xi+\xi_{0}\right) r_{1} \epsilon^{2} \operatorname{csch}^{4}\left(\frac{1}{2}\left(\xi+\xi_{0}\right) \sqrt{r_{1}}\right)}{4\left(\epsilon \operatorname{coth}\left(\frac{1}{2}\left(\xi+\xi_{0}\right) \sqrt{r_{1}}\right)+1\right)^{2}} \\
& +\frac{\left(\xi+\xi_{0}\right) \sqrt{r_{3}} r_{1}^{3 / 2} \epsilon \operatorname{csch}^{2}\left(\frac{1}{2}\left(\xi+\xi_{0}\right) \sqrt{r_{1}}\right)}{2 r_{2}}- \\
& \frac{\left(\xi+\xi_{0}\right) r_{1} \epsilon \operatorname{coth}\left(\frac{1}{2}\left(\xi+\xi_{0}\right) \sqrt{r_{1}}\right) \operatorname{csch}^{2}\left(\frac{1}{2}\left(\xi+\xi_{0}\right) \sqrt{r_{1}}\right)}{2\left(\epsilon \operatorname{coth}\left(\frac{1}{2}\left(\xi+\xi_{0}\right) \sqrt{r_{1}}\right)+1\right)},
\end{aligned}
$$

$r_{1}>0, r_{2}^{2}-4 r_{1} r_{3}=0$.

\section{Case - II}

$v_{2}=$

$$
\begin{aligned}
& -\frac{\sqrt{\frac{r_{1}}{r_{3}}}\left(\frac{\sqrt{r_{1}} \epsilon \cosh \left(\left(\xi+\xi_{0}\right) \sqrt{r_{1}}\right)}{\eta+\cosh \left(\left(\xi+\xi_{0}\right) \sqrt{r_{1}}\right)}-\frac{\sqrt{r_{1}} \epsilon \sinh ^{2}\left(\left(\xi+\xi_{0}\right) \sqrt{r_{1}}\right)}{\left(\eta+\cosh \left(\left(\xi+\xi_{0}\right) \sqrt{r_{1}}\right)\right)^{2}}\right)}{2\left(\sqrt{\frac{r_{1}}{4 r_{3}}}\left(\frac{\epsilon \sinh \left(\left(\xi+\xi_{0}\right) \sqrt{r_{1}}\right)}{\eta+\cosh \left(\left(\xi+\xi_{0}\right) \sqrt{r_{1}}\right)}+1\right)\right)} \\
& +A_{0} \\
& -\sqrt{\frac{r_{1}}{4}}\left(\frac{\epsilon \sinh \left(\left(\xi+\xi_{0}\right) \sqrt{r_{1}}\right)}{\eta+\cosh \left(\left(\xi+\xi_{0}\right) \sqrt{r_{1}}\right)}+1\right),
\end{aligned}
$$




$$
\begin{aligned}
r_{1} & >0, r_{3}>0, r_{2}=\sqrt{4 r_{1} r_{3}} \\
u_{2}= & \left(\frac{\sqrt{r_{1}} \epsilon \cosh \left(\left(\xi+\xi_{0}\right) \sqrt{r_{1}}\right)}{\eta+\cosh \left(\left(\xi+\xi_{0}\right) \sqrt{r_{1}}\right)}\right. \\
- & \frac{\sqrt{r_{1}} \epsilon \sinh ^{2}\left(\left(\xi+\xi_{0}\right) \sqrt{r_{1}}\right)}{\left(\eta+\cosh \left(\left(\xi+\xi_{0}\right) \sqrt{r_{1}}\right)\right)^{2}} \frac{\left(\xi+\xi_{0}\right) \sqrt{r_{1}} \epsilon \cosh \left(\sqrt{r_{1}} x\right)}{\eta+\cosh \left(\sqrt{r_{1}}\right)} \\
- & \left.\frac{\left(\xi+\xi_{0}\right) \sqrt{r_{1}} \epsilon \sinh ^{2}\left(\sqrt{r_{1}}\right)}{\left(\eta+\cosh \left(\sqrt{r_{1}}\right)\right)^{2}}\right) \\
/ & \left(\frac{\epsilon \sinh \left(\left(\xi+\xi_{0}\right) \sqrt{r_{1}}\right)}{\eta+\cosh \left(\sqrt{r_{1}}\right)}+1\right)^{2} \\
- & \frac{1}{2} \sqrt{r_{1}}\left(\frac{\left(\xi+\xi_{0}\right) \sqrt{r_{1}} \epsilon \cosh \left(\sqrt{r_{1}}\right)}{\eta+\cosh \left(\left(\xi+\xi_{0}\right) \sqrt{r_{1}}\right)}\right. \\
- & \left.\frac{\left(\xi+\xi_{0}\right) \sqrt{r_{1}} \epsilon \sinh { }^{2}\left(\sqrt{r_{1}}\right)}{\left(\eta+\cosh \left(\left(\xi+\xi_{0}\right) \sqrt{r_{1}}\right)\right)^{2}}\right) \\
- & \frac{1}{{ }^{2} \sinh \left(\sqrt{r_{1}} x\right)} \frac{2\left(\xi+\xi_{0}\right) r_{1} \epsilon \sinh ^{3}\left(\sqrt{r_{1}}\right)}{\left(\eta+\cosh \left(\left(\xi+\xi_{0}\right) \sqrt{r_{1}}\right)\right)^{3}} \\
+ & \frac{r_{1} \epsilon \sinh \left(\sqrt{r_{1}}\right)}{\eta+\cosh \left(\sqrt{r_{1}}\right)} \\
- & \frac{3\left(\xi+\xi_{0}\right) r_{1} \epsilon \sinh \left(\left(\xi+\xi_{0}\right) \sqrt{\left.r_{1}\right)} \cosh \left(\sqrt{r_{1}}\right)\right.}{\left(\eta+\cosh \left(\sqrt{r_{1}}\right)\right)^{2}} \\
r_{1}> & 0, r_{3}>0, r_{2}=\sqrt{4 r_{1} r_{3}}
\end{aligned}
$$

\section{Case - III}

$$
\begin{gathered}
v_{3}=-\frac{r_{1}\left(\frac{\sqrt{r_{1}} \epsilon \cosh \left(\left(\xi+\xi_{0}\right) \sqrt{r_{1}}\right)}{\eta \sqrt{p^{2}+1}+\cosh \left(\left(\xi+\xi_{0}\right) \sqrt{r_{1}}\right)}\right.}{\frac{r_{2}\left(r_{1}\left(\frac{\epsilon\left(p+\sinh \left(\left(\xi+\xi_{0}\right) \sqrt{r_{1}}\right)\right)}{\eta \sqrt{p^{2}+1}+\cosh \left(\left(\xi+\xi_{0}\right) \sqrt{r_{1}}\right)}+1\right)\right)}{r_{2}}} \\
-\frac{\left.\frac{\sqrt{r_{1}} \epsilon \sinh \left(\left(\xi+\xi_{0}\right) \sqrt{r_{1}}\right)\left(p+\sinh \left(\left(\xi+\xi_{0}\right) \sqrt{r_{1}}\right)\right)}{\left(\eta \sqrt{p^{2}+1}+\cosh \left(\left(\xi+\xi_{0}\right) \sqrt{r_{1}}\right)\right)^{2}}\right)}{\frac{r_{2}\left(r_{1}\left(\frac{\epsilon\left(p+\sinh \left(\left(\xi+\xi_{0}\right) \sqrt{r_{1}}\right)\right)}{\left.\eta \sqrt{p^{2}+1}+\cosh \left(\xi \xi+\xi_{0}\right) \sqrt{r_{1}}\right)}+1\right)\right)}{r_{2}}}+ \\
A_{0}+\frac{r_{1} \sqrt{r_{3}\left(\frac{\epsilon\left(p+\sinh \left(\left(\xi+\xi_{0}\right) \sqrt{r_{1}}\right)\right)}{\eta \sqrt{p^{2}+1}+\cosh \left(\left(\xi+\xi_{0}\right) \sqrt{r_{1}}\right)}+1\right)}}{r_{2}}, \\
r_{1}>0
\end{gathered}
$$$$
u_{3}=\frac{r_{1} \sqrt{r_{3}}\left(\frac{\left(\xi+\xi_{0}\right) \sqrt{r_{1}} \epsilon \cosh \left(\left(\xi+\xi_{0}\right) \sqrt{r_{1}}\right)}{\eta \sqrt{p^{2}+1}+\cosh \left(\left(\xi+\xi_{0}\right) \sqrt{r_{1}}\right)}\right.}{r_{2}}
$$

$$
\begin{aligned}
& \sqrt{r_{1}}\left(\sqrt{r_{1}} \epsilon \cosh \left(\left(\xi+\xi_{0}\right) \sqrt{r_{1}}\right)+\sqrt{r_{1}} \sinh \left(\sqrt{r_{1}}\right)\right) \\
& \left(\eta \sqrt{p^{2}+1} \epsilon \cosh \left(\left(\xi+\xi_{0}\right) \sqrt{r_{1}}\right)\right. \\
& \left.-\epsilon \mathrm{p} \sinh \left(\left(\xi+\xi_{0}\right) \sqrt{r_{1}}\right)+\epsilon\right) \\
& /\left(\eta \sqrt{p^{2}+1}+\cosh \left(\left(\xi+\xi_{0}\right) \sqrt{r_{1}} x\right)\right)\left(\eta \sqrt{p^{2}+1}\right. \\
& \left.+\epsilon\left(p+\sinh \left(\left(\xi+\xi_{0}\right) \sqrt{r_{1}}\right)\right)+\cosh \left(\left(\xi+\xi_{0}\right) \sqrt{r_{1}}\right)\right)^{2} \\
& +\sqrt{r_{1}} \epsilon\left(\eta\left(\xi+\xi_{0}\right) \sqrt{p^{2}+1} \sqrt{r_{1}} \sinh \left(\left(\xi+\xi_{0}\right) \sqrt{r_{1}}\right)\right. \\
& \left.-\left(\xi+\xi_{0}\right) p \sqrt{r_{1}} \cosh \left(\sqrt{r_{1}}\right)\right) /\left(\eta \sqrt{p^{2}+1}+\cosh \left(\sqrt{r_{1}}\right)\right) \\
& \eta \sqrt{p^{2}+1}+\epsilon\left(p+\sinh \left(\left(\xi+\xi_{0}\right) \sqrt{r_{1}}\right)\right) \\
& \left.+\cosh \left(\left(\xi+\xi_{0}\right) \sqrt{r_{1}}\right)\right), r_{1}>0
\end{aligned}
$$

\subsection{Applications of Extended Simple Equation Method:}

Let (12) has solution,

$$
v=A_{1} \Psi+\frac{A_{-1}}{\Psi}+A_{0}
$$

Put (21) in (12) along with (7) and after solving obained system of equations, we have

Case I: $l_{3}=0$,

\section{Family - I}

$$
A_{1}=-2 l_{2}, \quad A_{-1}=0, \quad \omega=l_{1}^{2}-4 l_{0} l_{2}
$$

Substitute (22) in (21) with (7), then solution of Eq. (10) achieved,

$$
\begin{aligned}
& v_{4}=A_{0} \\
&+\left(l_{1}-\sqrt{4 l_{0} l_{2}-l_{1}^{2}} \tan \left(\frac{\sqrt{4 l_{0} l_{2}-l_{1}^{2}}}{2}\left(\xi+\xi_{0}\right)\right)\right) \\
& 4 l_{0} l_{2}>l_{1}^{2} \\
& u_{4}=-\frac{1}{2}\left(4 l_{0} l_{2}\right. \\
&\left.-l_{1}^{2}\right)\left(\xi+\xi_{0}\right) \sec ^{2}\left(\frac{1}{2} \sqrt{4 l_{0} l_{2}-l_{1}^{2}}\left(\xi+\xi_{0}\right)\right), 4 l_{0} l_{2}>l_{1}^{2}
\end{aligned}
$$

$$
\begin{aligned}
& \left.\quad \frac{\left(\xi+\xi_{0}\right) \sqrt{r_{1}} \epsilon \sinh \left(\left(\xi+\xi_{0}\right) \sqrt{r_{1}}\right)\left(p+\sinh \left(\left(\xi+\xi_{0}\right) \sqrt{r_{1}}\right)\right)}{\left(\eta \sqrt{p^{2}+1}+\cosh \left(\left(\xi+\xi_{0}\right) \sqrt{r_{1}}\right)\right)^{2}}\right) \\
& -\frac{r_{2}}{+} \\
& +\left(\xi+\xi_{0}\right) r_{1} \epsilon \sinh \left(\left(\xi+\xi_{0}\right) \sqrt{r_{1}}\right)\left(\eta \sqrt{p^{2}+1} \cosh ((\xi\right. \\
& \left.\left.\left.+\xi_{0}\right) \sqrt{r_{1}} x\right)-p \sinh \left(\left(\xi+\xi_{0}\right) \sqrt{r_{1}}\right)+1\right)
\end{aligned}
$$

\section{Family - II}

$$
A_{1}=0, \quad A_{-1}=2 l_{0}, \quad \omega=l_{1}^{2}-4 l_{0} l_{2}
$$



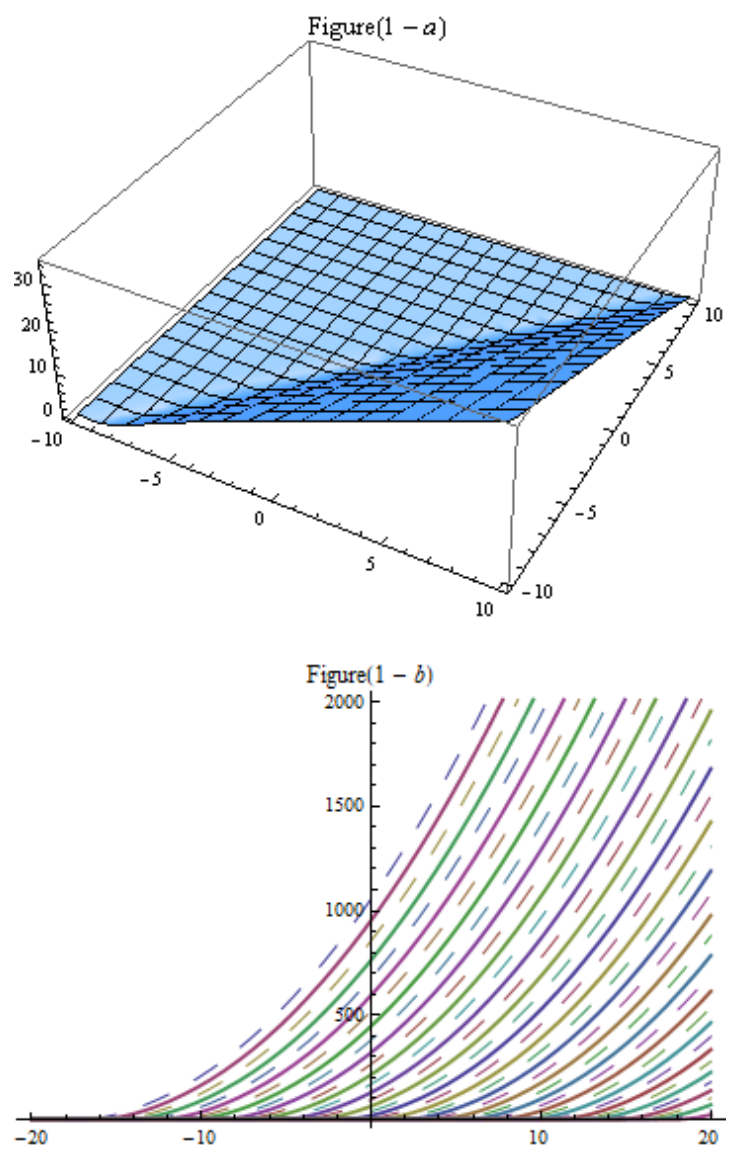

Figure 1: Exact traveling waves of solution (20).

Put (25) in (21),

$v_{5}=A_{0}$

(26)

$$
-\frac{4 l_{2} l_{0}}{\left(l_{1}-\sqrt{4 l_{2} l_{0}-l_{1}^{2}} \tan \left(\frac{1}{2} \sqrt{4 l_{2} l_{0}-l_{1}^{2}}\left(\xi+\xi_{0}\right)\right)\right)},
$$

$4 l_{0} l_{2}>l_{1}^{2}$.

$$
u_{5}=\frac{2 l_{0} l_{2}\left(4 l_{0} l_{2}-l_{1}^{2}\right)\left(\xi+\xi_{0}\right) \sec ^{2}\left(\frac{1}{2} \sqrt{4 l_{0} l_{2}-l_{1}^{2}}\left(\xi+\xi_{0}\right)\right)}{\left(l_{1}-\sqrt{4 l_{0} l_{2}-l_{1}^{2}} \tan \left(\frac{1}{2} \sqrt{4 l_{0} l_{2}-l_{1}^{2}}\left(\xi+\xi_{0}\right)\right)\right)^{2}},
$$$$
4 l_{0} l_{2}>l_{1}^{2}
$$

Case II: $l_{0}=l_{3}=0$,

$$
\omega=l_{1}^{2}, \quad A_{1}=-2 l_{2}, \quad A_{-1}=0
$$

Put (28) in (21),

$$
\begin{gathered}
v_{6}=\frac{-2 l_{2} l_{1} e^{l_{1}\left(\xi+\xi_{0}\right)}}{\left(1-l_{2} e^{l_{1}\left(\xi+\xi_{0}\right)}\right)}, \quad l_{1}>0 . \\
u_{6}=-\frac{2 l_{1}^{2} l_{2}^{2}\left(\xi+\xi_{0}\right) e^{l_{1}\left(\xi+\xi_{0}\right)}}{\left(1-l_{2} e^{l_{1}\left(\xi+\xi_{0}\right)}\right)^{2}}, \quad l_{1}>0 .
\end{gathered}
$$
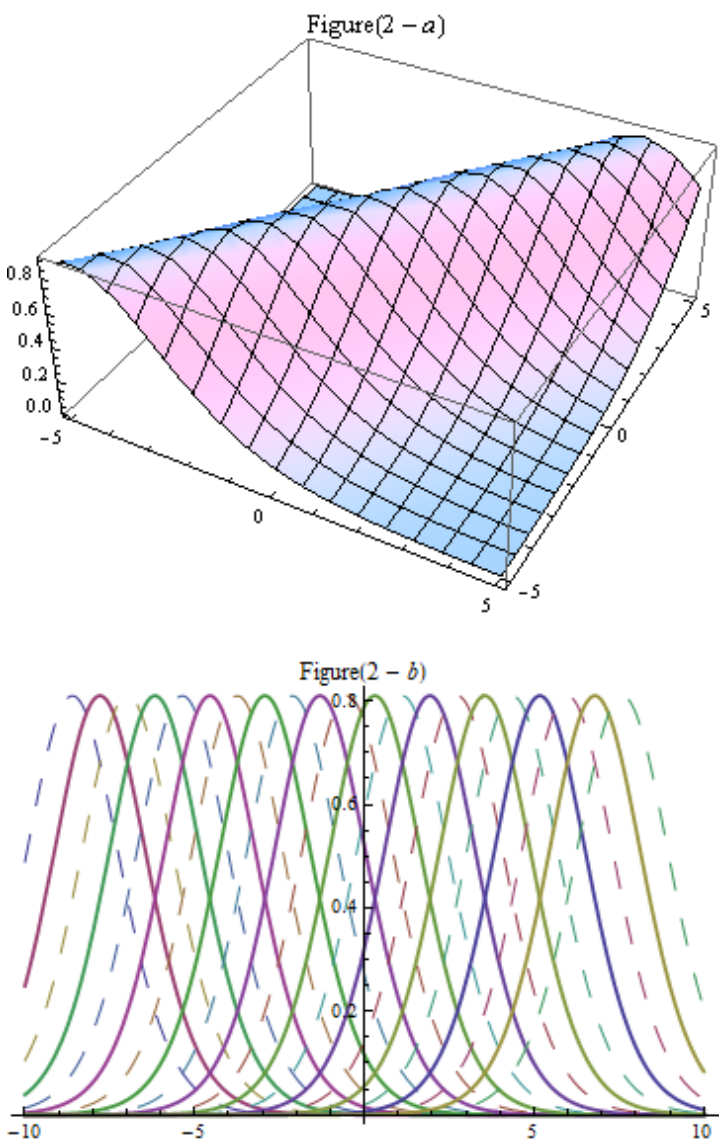

Figure 2: Traveling waves of solution of (30).

$$
v_{7}=\frac{2 l_{2} l_{1} e^{l_{1}\left(\xi+\xi_{0}\right)}}{\left(1+l_{2} e^{l_{1}\left(\xi+\xi_{0}\right)}\right)}, \quad l_{1}<0
$$

$$
u_{7}=-\frac{2 l_{1}^{2} l_{2}^{2}\left(\xi+\xi_{0}\right) e^{l_{1}\left(\xi+\xi_{0}\right)}}{\left(l_{2} e^{l_{1}\left(\xi+\xi_{0}\right)}+1\right)^{2}}, \quad l_{1}<0 .
$$

Case III: $l_{1}=l_{3}=0$,

\section{Family - I}

$$
\omega=-4 l_{0} l_{2}, \quad A_{1}=-2 l_{2}, \quad A_{-1}=0
$$

Put (33) in (21),

$$
v_{8}=A_{0}-2 \sqrt{l_{0} l_{2}}\left(\tan \sqrt{l_{0} l_{2}}\left(\xi+\xi_{0}\right)\right), \quad l_{2} l_{0}>0 .
$$

$$
u_{8}=-2 l_{0} l_{2}\left(\xi+\xi_{0}\right) \sec ^{2}\left(\sqrt{l_{0} l_{2}}\left(\xi+\xi_{0}\right)\right),
$$

$l_{2} l_{0}>0$.

$$
v_{9}=A_{0}+2 \sqrt{-l_{0} l_{2}}\left(\tanh \sqrt{-l_{0} l_{2}}\left(\xi+\xi_{0}\right)\right),
$$

$l_{2} l_{0}<0$. 

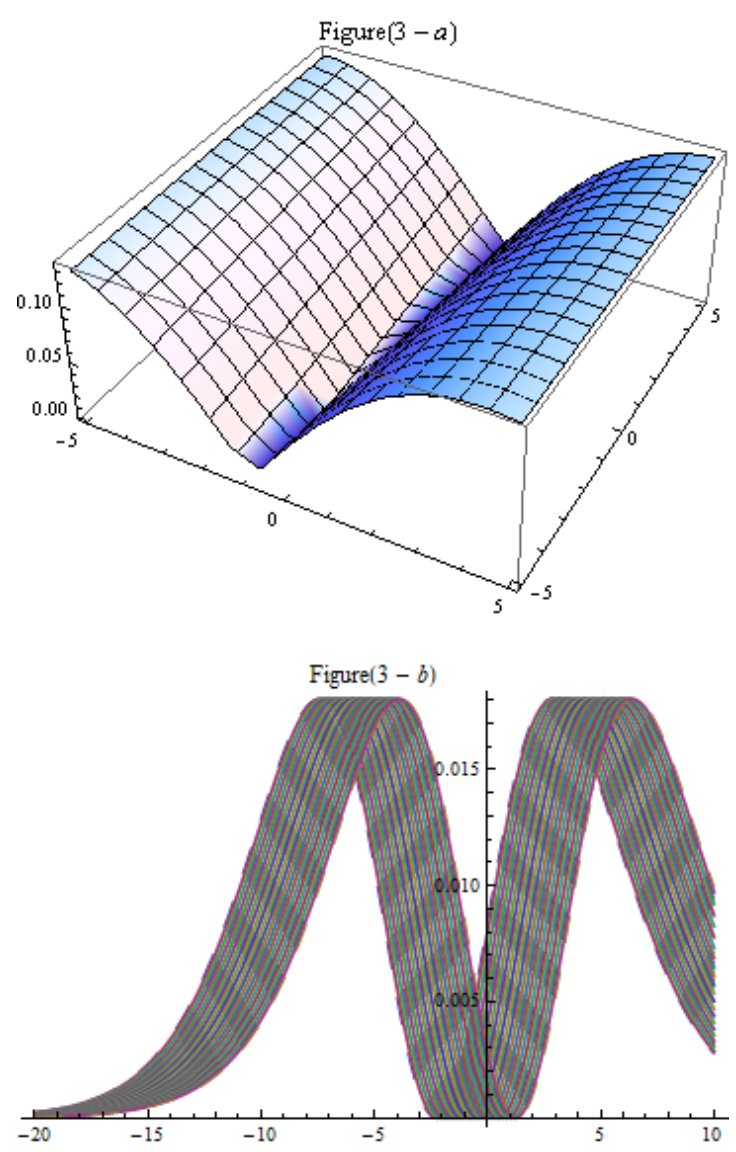

Figure 3: Traveling waves of solution (32).

$$
\begin{aligned}
& u_{9}=-2 l_{0} l_{2}\left(\xi+\xi_{0}\right) \operatorname{sech}^{2}\left(\sqrt{-l_{0} l_{2}}\left(\xi+\xi_{0}\right)\right), \\
& l_{2} l_{0}<0 .
\end{aligned}
$$

\section{Family - II}

$$
\omega=-4 l_{0} l_{2}, \quad A_{1}=0, \quad A_{-1}=2 l_{0}
$$

Put (38) in (21),

$$
\begin{gathered}
v_{10}=A_{0}-\frac{2 l_{0} l_{2}}{\sqrt{l_{0} l_{2}}\left(\tan \sqrt{l_{0} l_{2}}\left(\xi+\xi_{0}\right)\right)}, \quad l_{0} l_{2}>0, \\
u_{10}=-2 l_{0} l_{2}\left(\xi+\xi_{0}\right) \csc ^{2}\left(\sqrt{l_{0} l_{2}}\left(\xi+\xi_{0}\right)\right), \\
l_{0} l_{2}>0,
\end{gathered}
$$$$
v_{11}=A_{0}+\frac{2 l_{0} l_{2}}{\left(\sqrt{-l_{0} l_{2}} \tanh \sqrt{-l_{0} l_{2}}\left(\xi+\xi_{0}\right)\right)},
$$$$
l_{0} l_{2}<0,
$$$$
u_{11}=2 l_{0} l_{2}\left(\xi+\xi_{0}\right) \operatorname{csch}^{2}\left(\sqrt{-l_{0} l_{2}}\left(\xi+\xi_{0}\right)\right),
$$$$
l_{0} l_{2}<0 \text {, }
$$

When $A=0, B=-1, C=1$, then we have,

$$
\omega=1, \quad a_{1}=-2, \quad b_{1}=0
$$

Substitute (52) into (48),

$$
\begin{gathered}
v_{15}=a_{0}-\left(1-\operatorname{coth}\left(\frac{1}{2} \xi\right)\right) \\
u_{15}=\frac{1}{2} \xi \operatorname{csch}^{2}\left(\frac{\xi}{2}\right)
\end{gathered}
$$

For $A=\frac{1}{2}, B=0, C=-\frac{1}{2}$, then we have, 

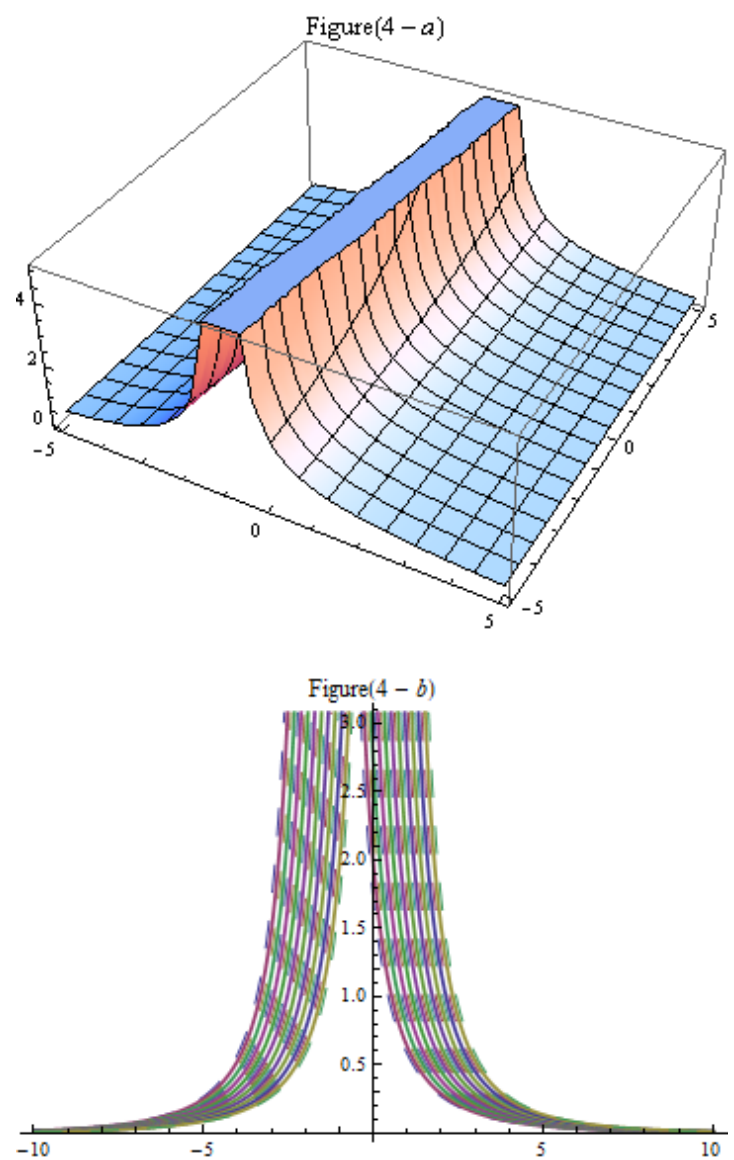

Figure 4: Traveling waves of solution of (42).

\section{Family - I}

$$
\omega=1, \quad a_{1}=0, \quad b_{1}=1
$$

Put (55) in (48),

$$
\begin{gathered}
v_{16}=a_{0}+\left(\frac{1}{\operatorname{coth}(\xi) \pm \operatorname{csch}(\xi)}\right) \\
u_{16}=-\frac{-\xi \operatorname{csch}^{2}(\xi)-\xi \operatorname{coth}(\xi) \operatorname{csch}(\xi)}{(\operatorname{coth}(\xi)+\operatorname{csch}(\xi))^{2}}
\end{gathered}
$$

\section{Family - II}

$$
\omega=-1, \quad a_{1}=1, \quad b_{1}=0
$$

Put (58) in (48),

$$
\begin{gathered}
v_{17}=a_{0}+( \pm \operatorname{csch}(\xi)+\operatorname{coth}(\xi)) \\
u_{17}=-\xi \operatorname{csch}^{2}(\xi)-\xi \operatorname{coth}(\xi) \operatorname{csch}(\xi)
\end{gathered}
$$

Family - III

$$
\omega=-4, \quad a_{1}=1, \quad b_{1}=-1
$$
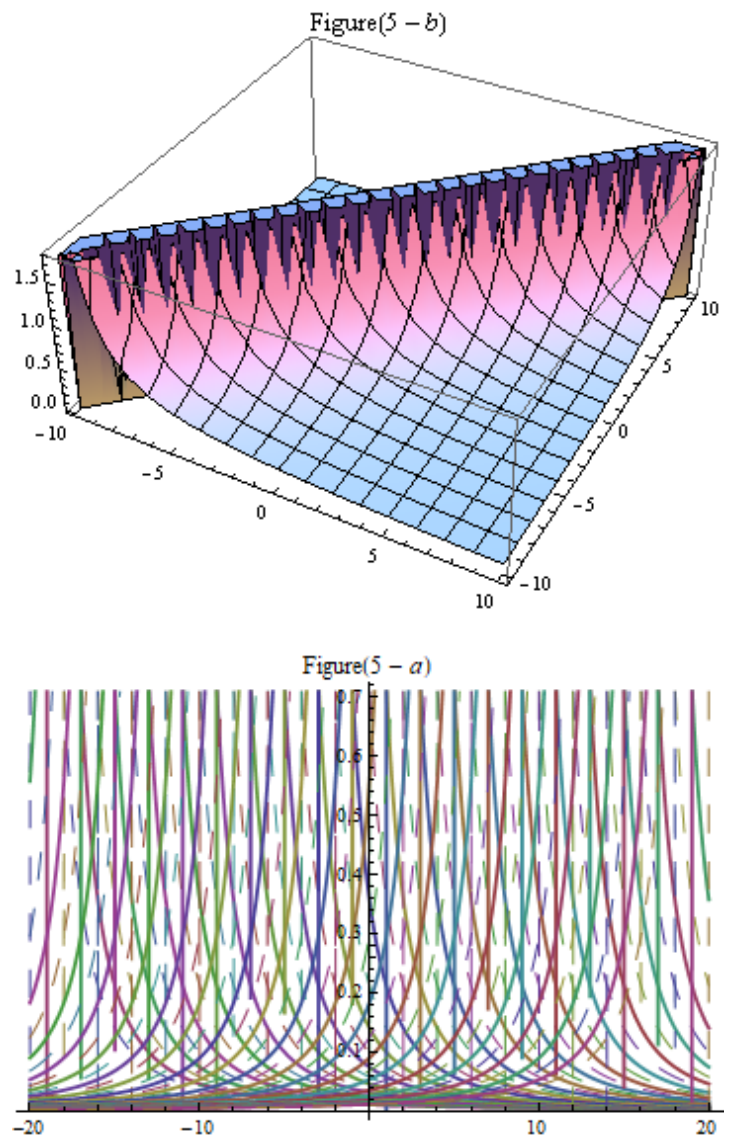

Figure 5: Traveling waves of solution (102).

Put (61) in (48),

$$
\begin{gathered}
v_{18}=a_{0}+\left(\frac{1}{( \pm \operatorname{csch}(\xi)+\operatorname{coth}(\xi))}\right) \\
+( \pm \operatorname{csch}(\xi)+\operatorname{coth}(\xi)) \\
u_{18}=-\xi \operatorname{csch}^{2}(\xi)-\frac{-\xi \operatorname{csch}^{2}(\xi)-\xi \operatorname{coth}(\xi) \operatorname{csch}(\xi)}{(\operatorname{coth}(\xi)+\operatorname{csch}(\xi))^{2}} \\
-\xi \operatorname{coth}(\xi) \operatorname{csch}(\xi)
\end{gathered}
$$

For $C=-1, B=0, A=1$,

\section{Family - I}

$$
\omega=4, \quad a_{1}=0, \quad b_{1}=2
$$

Put (64) in (48),

$$
v_{19}=a_{0}+2\left(\frac{1}{\tanh (\xi)}\right), \quad \text { or } \quad a_{0}+2\left(\frac{1}{\operatorname{coth}(\xi)}\right)
$$

$$
u_{19}=-\xi \operatorname{csch}^{2}(\xi), \quad \text { or } \quad \xi \operatorname{sech}^{2}(\xi)
$$

Family - II

$$
\omega=4, \quad a_{1}=2, \quad b_{1}=0
$$


Put (67) in (48),

$$
\begin{array}{r}
v_{20}(\xi)=a_{0}+2(\tanh (\xi)) \text { or } a_{0}+(\operatorname{coth}(\xi)) \\
u_{20}(\xi)=\xi \operatorname{sech}^{2}(\xi) \text { or }-\xi \operatorname{csch}^{2}(\xi)
\end{array}
$$

\section{Family - III}

$$
\omega=16, \quad a_{1}=2, \quad b_{1}=2
$$

Put (70) in (29),

$$
\begin{gathered}
v_{21}=a_{0}+2\left(\tanh (\xi)+\frac{1}{\tanh (\xi)}\right) \\
\text { or } a_{0}+2\left(\operatorname{coth}(\xi)+\frac{1}{\operatorname{coth}(\xi)}\right) \\
u_{21}=2 \xi \operatorname{sech}^{2}(\xi)-\xi \operatorname{csch}^{2}(\xi) \\
\text { or } 2 \xi \operatorname{sech}^{2}(\xi)-\xi \operatorname{csch}^{2}(\xi)
\end{gathered}
$$

When $A=\frac{1}{2}, C=\frac{1}{2}, B=0$,

\section{Family - I}

$$
\omega=-1, \quad a_{1}=-1, \quad b_{1}=0
$$

Put (73) in (48),

$$
\begin{gathered}
v_{22}=a_{0}-(\sec (\xi)+\tan (\xi)) \\
u_{22}=\xi \sec ^{2}(\xi)+\xi \tan (\xi) \sec (\xi)
\end{gathered}
$$

\section{Family - II}

$$
\omega=-1, \quad a_{1}=0, \quad b_{1}=1
$$

Put (76) in (48),

$$
\begin{gathered}
v_{23}=a_{0}+\left(\frac{1}{\tan (\xi)+\sec (\xi)}\right) \\
u_{23}=-\frac{\xi \sec ^{2}(\xi)+\xi \tan (\xi) \sec (\xi)}{(\tan (\xi)+\sec (\xi))^{2}}
\end{gathered}
$$

\section{Family - III}

$$
\omega=-4, \quad a_{1}=-1, \quad b_{1}=1
$$

By putting Eq. (79) in (48),

$$
\begin{aligned}
v_{24}= & a_{0}-(\tan (\xi)+\sec (\xi))+\left(\frac{1}{\tan (\xi)+\sec (\xi)}\right) \\
u_{24} & =\xi \sec ^{2}(\xi)+\frac{\xi \sec ^{2}(\xi)+\xi \tan (\xi) \sec (\xi)}{(\tan (\xi)+\sec (\xi))^{2}} \\
& +\xi \tan (\xi) \sec (\xi) \\
A=-\frac{1}{2}, B & =0, C=-\frac{1}{2},
\end{aligned}
$$

\section{Family - I}

$$
\omega=-1, \quad a_{1}=1, \quad b_{1}=0
$$

Put (82) in (48),

$$
v_{25}=a_{0}+(\sec (\xi)-\tan (\xi))
$$

$$
u_{25}=\xi \tan (\xi) \sec (\xi)-\xi \sec ^{2}(\xi)
$$

\section{Family - II}

$$
\omega=-1, \quad a_{1}=0, \quad b_{1}=-1
$$

Put (85) in (48),

$$
\begin{gathered}
v_{26}=a_{0}-\left(\frac{1}{\tan (\xi)-\sec (\xi)}\right) \\
u_{26}=\frac{\xi \sec ^{2}(\xi)-\xi \tan (\xi) \sec (\xi)}{(\tan (\xi)-\sec (\xi))^{2}}
\end{gathered}
$$

\section{Family - III}

$$
\omega=-4, \quad a_{1}=1, \quad b_{1}=-1
$$

Put (88) in (48),

$$
v_{27}=a_{0}+(\sec (\xi)-\tan (\xi))-\frac{3}{2}\left(\frac{1}{\tan (\xi)-\sec (\xi)}\right)
$$

$$
\begin{aligned}
u_{27} & =-\xi \sec ^{2}(\xi)-\frac{\xi \tan (\xi) \sec (\xi)-\xi \sec ^{2}(\xi)}{(\sec (\xi)-\tan (\xi))^{2}} \\
& -\xi \tan (\xi) \sec (\xi)
\end{aligned}
$$

$C=A=-1, B=0$,

\section{Family - I}

$$
\omega=-4, \quad a_{1}=2, \quad b_{1}=0
$$

Put (91) in (48),

$$
v_{28}=a_{0}+2(\tan (\xi)), \quad \text { or } \quad a_{0}+2(\cot (\xi))
$$

$$
u_{28}=2 \xi \sec ^{2}(\xi), \quad \text { or } \quad-2 \xi \csc ^{2}(\xi)
$$

\section{Family - II}

$$
\omega=-4, \quad a_{1}=0, \quad b_{1}=-2
$$

Put (94) in (48),

$$
\begin{gathered}
v_{29}=a_{0}-2\left(\frac{1}{(\tan (\xi)}\right), \text { or } a_{0}-2\left(\frac{1}{(\cot (\xi)}\right) \\
u_{29}=2 \xi \csc ^{2}(\xi x), \text { or }-2 \xi \sec ^{2}(\xi)
\end{gathered}
$$




\section{Family - III}

$$
\omega=-16, \quad a_{1}=2, \quad b_{1}=-2
$$

Put (97) in (48),

$$
\begin{gathered}
v_{30}(x, t)=a_{0}+2\left(\frac{1}{(\tan (\xi)}\right)-2(\tan (\xi)) \\
u_{30}(x, t)=-2 \xi \csc ^{2}(\xi)-2 \xi \sec ^{2}(\xi)
\end{gathered}
$$

When $A=0, B=1, C_{3} \neq 0$, then we have,

$$
\omega=1, \quad a_{1}=-2 C, \quad b_{1}=0
$$

Put (100) in (48),

$$
\begin{gathered}
v_{31}=a_{0}+2 C\left(\frac{1}{C \xi+\epsilon)}\right) \\
u_{31}=-\frac{2 C^{2} \xi}{(C \xi+\epsilon)^{2}}
\end{gathered}
$$

When $B=0, C=0$, then we have,

$$
a_{1}=\frac{\omega}{3 A}, \quad b_{1}=0
$$

Put (103) in (48),

$$
\begin{gathered}
v_{32}=\frac{\omega \xi}{3} \\
u_{32}=\frac{1}{3}(\omega \xi)
\end{gathered}
$$

When $A \neq 0, B \neq 0, C=0$, then we have,

$$
\omega=B^{2}, \quad a_{1}=0, \quad b_{1}=2 A
$$

Put (106) in (48),

$$
\begin{gathered}
v_{33}=a_{0}+2 A\left(\frac{B}{(\exp (B \xi)-A)}\right) \\
u_{33}=-\frac{2 A B^{2} \xi e^{B \xi}}{\left(e^{B \xi}-A\right)^{2}}
\end{gathered}
$$

\section{Results and Discussion}

Different researchers used distinct schemes for the determination of solutions of integro-differential Ito model [39, 40]. But here we have investigated serval types solutions nonlinear Eq. (12) via three analytical modified mathematical mathematical methods. With different values of the parameters in Eq. (4), Eq. (6) and Eq. (6) respectively obtained many different types solutions. However, some our investigated results are likely similar to with other researchers results in $[39,40]$. Our solution (30) and(32) are approximate similar to the solutions (18) and (21) in [39]. Solution (18) and (20) likely similar to (3.17) and (3.18) in [40].

Figure 1-5 are plotted after assigning these particular values to the parameters such that, solution $u_{3}(x, t)$ at $\eta=1, p=-1, r_{1}=0.9, r_{2}=2 r_{3}=5, \xi_{0}=0.07, \epsilon=-1$, $\omega=r_{1}$ and $u_{6}(x, t)$ at $4 l_{0}=1, l_{1}=0.9, l_{2}=1 \epsilon=0.5$ and $u_{7}(x, t)$ at $l_{0}=1, l_{1}=-0.3, l_{2}=1, \epsilon=0.5, \omega=l_{1}^{2}$ and $u_{11}(x, t) l_{0}=0.05, l_{2}=-0.5, \epsilon=0.5, \omega=-4 l_{0} l_{2}$ and $u_{31}$ at $B=6, \omega=1, \epsilon=1$ respectively. From results discussion and graphical representations of $u_{3}, u_{6}, u_{7}, u_{11} u_{31}$ by assigning the particular values with the assistance of Mathematica sofware, we have found that our techniques provide a rich plate form as a mathematical tools for solving nonlinear wave problem in Mathematics, physics and engineerings.

\section{Conclusion}

In this work, three analytical modified mathematical methods so called generalized direct algebraic, extended simplest equation and modified F-expansion methods are serve for the construction of the wave solutions of integrodifferential Ito equation, having important applications in mathematical physics. The investigated results are more general and provide a basic ground for solving many nonlinear problems.

\section{References}

[1] Inc M, Isa A, Yusuf A, Baleanu D. New solitary wave solutions and conservation laws to the Kudryashov-Sinelshchikov equation. Optik (Stuttg). 2017;142:665-73.

[2] Hassan A. New Exact Solutions for the Maccari System. J Phys. 2018.

[3] Bianca C, Pappalardo F, Motta S, Ragusa MA. Persistence analysis in a Kolmogorov- type model for cancer-immune system competition. AIP Conf Proc. 2013;1558:1797-800.

[4] Gala S, Guo Z, Ragusa MA. A remark on the regularity criterion of Boussinesq equations with zero heat conductivity. Appl Math Lett. 2014;27:70-3.

[5] Gala S, Ragusa MA. Logarithmically improved regularity criterion for the Boussinesq equations in Besov spaces with negative indices. Appl Anal. 2016;95(6):1271-9.

[6] Xu X, Zhu N. Global well-posedness for the 2D Boussinesq equations with partial temperature- dependent dissipative terms. J Math Anal Appl. 2018;466(1):351-72. 
[7] Khan K, Akbar M, Mohd NH. The modified simple equation method for exact and solitary wave solutions of nonlinear evolution equation. ISRN Mathematical Physics; 2013.

[8] Arshad M, Seadawy AR, Lu D. Exact bright-dark solitary wave solutions of the higher-order cubic-quintic nonlinear schrodinger equation and its stability. Optik (Stuttg). 2017;138:1-14.

[9] Ali M. Exact solutions of the generalized $(2+1)$ - dimensional nonlinear evolution equations via the modified simple method. Comput Math Appl. 2015;69(5):390-7.

[10] Ul-Haq Tariq K, Seadawy A. Soliton solutions of $(3+1)$ Dimensional Korteweg-de Vries Benjamin-Bona-Mahony, Kadomtsev-Petviashvili Benjamin-Bona-Mahony and modified Korteweg de Vries-Zakharov-Kuznetsov equations and their applications in water waves. Journal of King Saud University Science. 2019;31(1):8-13.

[11] Arshad M, Seadawy AR. Dianchen. Lu, J. Wang, Modulation instability analysis of modify unstable nonlinear Schrdinger dynamical equation and its optical soliton solutions. Results Phys. 2017;7:4153-61.

[12] Arshad M, Seadawy AR, Lu D. Modulation stability and optical soliton solutions of nonlinear Schrdinger equation with higher order dispersion and nonlinear terms and its applications. Superlattices Microstruct. 2017;112:422-34.

[13] Abdullah, Seadawy AR, Jun W. Mathematical methods and solitary wave solutions of three- dimensional Zakharov-KuznetsovBurgers equation in dusty plasma and its applications. Results Phys. 2017;7:4269-77.

[14] Abdullah, Seadawy AR, Jun W. Modified KdVZakharovKuznetsov dynamical equation in a homogeneous magnetised electronpositron-ion plasma and its dispersive solitary wave solutions. Pramana .J. Phys, 2018.

[15] Fu T, Li Z, Qi D, Qing Z. Conservation laws, bright matter wave solitons and modulational in- stability of nonlinear schroedinger equation with time-dependent nonlinearity. Commun Nonlinear Sci Numer Simul. 2012;17(8):3247-57.

[16] Zhou ZJ, Fu JZ, Li ZB. Maple packages for computing Hirotas bilinear equation and multisoliton solutions of nonlinear evolution equations. Appl Math Comput. 2010;217(1):92-104.

[17] Qian Z, Lihua W. Lin. F, Darboux transformation and explicit solutions to the generalized TD equation. Appl Math. 2017;67:1-6.

[18] Fu S, Sheng Z, Jiang W, Qing ZH. Darboux transformation operators and supersymmetry for a generalized one-dimensional time-dependent Schr ödinger equation. Appl Math Comput. 2012;218:7308-21.

[19] Lu X, Xiu W, Shouting C, Khalique CM. A note on rational solutions to a Hirota-Satsuma- like equation. Appl Math. 2016;58:13-8.

[20] Yang L, Liu J, Yang K. Exact solutions of nonlinear PDE nonlinear transformations and re- duction of nonlinear PDE to a quadrature. Phys. 2001;278:267-70.

[21] Matveev VB, Salle AM. Darboux Transformation and Solitons. Springer; 1991. https://doi.org/10.1007/978-3-662-00922-2.

[22] Eslami M, Mirzazadeh M. First integral method to look for exact solutions of a variety of Boussinesq-like equations. Ocean Eng. 2014;83:133-7.

[23] Yuanfen X. Bifurcations of the exact traveling solutions for $(2+1)$-dimensional HMIS equation. Commum Theor Phys. 2012;57(1):68-70.
[24] Gorza SP, Haelterman M. Ultrafast transverse undulation of selftrapped laser beams. Opt Express. 2008 Oct;16(21):16935-40.

[25] Tan BK, Wu RS, Nonlinear Rossby waves and their interactions. I. Collision of envelope solitary Rossby waves. Sci. China, 1993, $36: 1367$

[26] Tang XY, Shukla PK. Lie symmetry analysis of the quantum Zakharov equations. Phys. Scr A. 2007;76(6):665-8.

[27] Khater AH, Callebaut DK, Seadawy AR. General Soliton Solutions for Nonlinear Dispersive Waves in Convective Type Instabilities. Phys Scr. 2006;74(3):384-93.

[28] Saha Ray S. New exact solutions of nonlinear fractional acoustic wave equations in ultra- sound. Comput Math Appl. 2016;71(3):859-68.

[29] Ehab S. Selima, Aly R. Seadawy and Xiaohua Yao, The nonlinear dispersive Davey-Stewartson system for surface waves propagation in shallow water and its stability. Eur Phys J Plus. 2016;131(12):425.

[30] Seadawy AR, El-Rashidy K. Dispersive Solitary wave solutions of Kadomtsev-Petviashivili and modified Kadomtsev-Petviashivili dynamical equations in unmagnetized dust plasma. Results Phys. 2018;8:1216-1222.

[31] Seadawy AR, Alamri SZ, Mathematical methods via the nonlinear two-dimensional water waves of Olver dynamical equation and its exact solitary wave solutions. Results Phys. 2018;8:286291.

[32] Arnous AH, Seadawy AR, Rubayyi T. Alqahtani, Anjan Biswas, Optical solitons with complex GinzburgLandau equation by modified simple equation method. Optik (Stuttg). 2017;144:475480.

[33] Seadawy AR, Lu D, Yue C. Travelling wave solutions of the generalized nonlinear fifth-order KdV water wave equations and its stability. Journal of Taibah University for Science. 2017;11(4):62333.

[34] Abdullah AS, Wang J. Mathematical methods and solitary wave solutions of three-dimensional Zakharov-Kuznetsov-Burgers equation in dusty plasma and its applications. Results Phys. 2017;7:4269-4277.

[35] Seadawy AR. Two-dimensional interaction of a shear flow with a free surface in a stratified fluid and its a solitary wave solutions via mathematical methods. Eur Phys J Plus. 2017;132(12):518.

[36] Seadawy AR, El-Rashidy K. Rayleigh-Taylor instability of the cylindrical flow with mass and heat transfer, The Pramana -. J Phys. 2016;87:20.

[37] Seadawy AR, Solitary wave solutions of tow-dimensional nonlinear Kadomtsev-Petviashvili dynamic equation in a dust acoustic plasmas, The Pramana - Journal of Physics 89 (2017) 49:1-11.

[38] Khater AH, Callebaut DK, Helal MA, Seadawy AR. Variational Method for the Nonlinear Dynamics of an Elliptic Magnetic Stagnation Line. Eur Phys J D. 2006;39(2):237-45.

[39] Seadawy AR. Three dimensionalweaklynonlinearshallowwaterwavesregimeanditstravelling wave solutions. Int J Comput Methods. 2018;15(03):1850017.

[40] Seadawy AR, Manafian J. New soliton solution to the longitudinal wave equation in a magneto-electro-elastic circular rod. Results Phys. 2018;8:1158-1167.

[41] Aasaraai A. The Application of Modified F-expansion Method Solving the Maccaris System. British Journal of Mathematics and Computer Science. 2015: 11(5):1-14. 